\title{
Cálculo da força propulsiva gerada pela mão e antebraço do nadador através da dinâmica computacional de fluidos
}

\author{
A.J. Silva ${ }^{1}$ \\ A.F. Rouboa ${ }^{2}$ \\ L. Leal 2 \\ J. Rocha ${ }^{2}$ \\ F.B. Alves ${ }^{3}$ \\ A.M. Moreira ${ }^{4}$ \\ V.M. Reis ${ }^{1}$ \\ J.P. Vilas Boas ${ }^{5}$
}

https://doi.org/10.5628/rpcd.05.03.288

\section{RESUMO}

A investigação da força propulsiva produzida pela mão e antebraço dos nadadores tem-se baseado em testes experimentais. No entanto, existem algumas dúvidas sobre a precisão e fiabili-dade destes cálculos. Este estudo teve dois objectivos: i) dar continuidade ao uso da Dinâmica Computacional de Fluidos (DCF) como uma nova metodologia de investigação na Natação; ii) aplicar a DCF no cálculo dos coeficientes de resistência $\left(\mathrm{C}_{\mathrm{R}}\right)$ e sustentação $\left(C_{S}\right)$ resultantes da simulação numérica do fluxo externo da mão e antebraço. Para este efeito, utilizaram-se três modelos bidimensionais de um escoamento em regime permanente. Um modelo frontal $\left(\theta=90^{\circ}, \Phi=0^{\circ}\right)$ e dois modelos laterais, um tendo o dedo polegar como bordo de ataque $\left(\theta=180^{\circ}\right.$, $\left.\Phi=0^{\circ}\right)$, e o outro tendo o dedo mindinho como bordo de ataque $\left(\theta=0^{\circ}, \Phi=0^{\circ}\right)$. O sistema de resolução de equações utilizado foi o das equações de Navier -Stokes, para fluidos não compressíveis. Os principais resultados, demonstraram que o $C_{R}$ foi o coeficiente que contribuiu mais para a propulsão, sendo constante para toda a amplitude de velocidades com um valor máximo de 1,16 . Com base nestes resultados, podemos concluir que: i) a DCF pode ser considerada uma nova metodologia susceptível de desenvolvimento para o cálculo de forças hidrodinâmicas em Natação (de facto neste estudo não se procedeu à respectiva validação, pelo que não se pode concluir pela sua validade); ii) nos três modelos estudados o $\mathrm{C}_{\mathrm{S}}$ parece ter uma importância secundária na geração de força propulsiva.

\author{
${ }^{1}$ Universidade de Trás-os-Montes e Alto Douro \\ Departamento de Ciências do Desporto, Vila Real \\ 2 Universidade de Trás-os-Montes e Alto Douro \\ Departamento de Engenharias \\ ${ }^{3}$ Universidade Técnica de Lisboa \\ Faculdade de Motricidade Humana \\ ${ }^{4}$ Instituto Politécnico de Santarém \\ Escola Superior de Desporto \\ ${ }^{5}$ Universidade do Porto, Faculdade de Desporto, Portugal
}

\begin{abstract}
Measurement of swimmer's hand/forearm propulsive forces generation using Computational Fluid Dynamics.

Propulsive force generated by swimmers hand/forearm has been studied through experimental tests. However, there are serious doubts as to whether forces quantified with these procedures are accurate enough to be meaningful. The main purpose of the present work was twofold: $i$ ) continuing the use of computational fluid dynamics (CFD) as a new tool in swimming research; ii) apply the CFD method in the calculation of drag and lift coefficients resulting from the numerical resolution equations of the flow around the swimmers hand/forearm. For these purposes three, two-dimensional, models of a right male hand/forearm were studied. A frontal model $\left(\theta=90^{\circ}, \Phi=0^{\circ}\right)$ and two lateral models, one with the thumb as leading edge $\left(\theta=180^{\circ}, \Phi=0^{\circ}\right)$, and the other with the litle finger as the leading edge $\left(\theta=0^{\circ}, \Phi=0^{\circ}\right)$. The governing system of equations considered was the incompressible Reynolds averaged NavierStokes equations. The main results reported that the drag coefficient was the coefficient that accounts more for propulsion, and was almost constant for the whole range of velocities, with a maximum value of $1,16\left(C_{d}=1,16\right)$. Through the results, we can conclude that: $\left.i\right)$ CFD can be considered an interesting new approach for hydrodynamic forces calculation in swimming research; ii) in the three models studied the lift coefficient may be of secondary importance in force generation.
\end{abstract}

Key Words: CFD, turbulence models, drag, lift, hydrodynamics. 


\section{INTRODUÇÃO}

A força propulsiva gerada pelo comple xo segmentar formado pela mão e antebraço tem sido estudada, desde a década de 60 , recorrendo a métodos experimentais $(1,2,3,7,8,9,10,11,12,13,14,15,16$, $17,18,19)$. Só recentemente se recorreu a técnicas numéricas, mediante a aplicação da Dinâmica Computacional de Fluidos (DCF). A razão pela qual é determinante o cálculo das forças propulsivas baseia-se no facto de o movimento na água ser altamente dependente da interacção do sujeito com o fluido envolvente. Desta forma, a explicação do movimento humano no conte xto aquático requer a medição destas forças de interacção, que actuam fundamentalmente nos segmentos responsáveis pela propulsão (mão e antebraço).

A quantificação da contribuição da braçada na propulsão, sem qualquer constrangimento mecânico que possa causar uma alteração na técnica de nado natural, tem sido, ao longo dos tempos, efectuada com base em métodos indirectos. Nestes, as forças são estimadas mediante a aplicação dos coeficientes de resistência $\left(C_{R}\right)$ e sustentação hidrodinâmica $\left(\mathrm{C}_{\mathrm{S}}\right)$, obtidos em estudos laboratoriais $(1,2,3,11$, $12,13,15,17,18,19)$, aos valores de orientação e velocidade dos segmentos, decorrentes das análises tridimensionais resultantes de filmagens vídeo dos movimentos subaquáticos $(7,8)$.

Schleihauf (15) utilizou uma aproximação em duas etapas na aplicação da metodologia experimental: i) cálculo da força propulsiva em modelos de resina da mão em laboratório, mediante a aplicação de procedimentos da investigação aeronáutica, gerando os valores dos coeficientes $\left(\mathrm{C}_{\mathrm{R}}\right.$ e $\left.\mathrm{C}_{\mathrm{S}}\right)$, para uma ampla variedade de condições de fluxo; ii) aplicação destes coeficientes a movimentos reais decorrentes das análises do movimento. Berger, Groot \& Hollander (1), mediram os valores dos coeficientes $\left(C_{R}\right.$ e $\left.C_{S}\right)$ em dois modelos (resina) de uma mão e antebraço imersos num tanque de experimentação. De forma a comparar os resultados dos dois modelos para diferentes velocidades, os valores de força foram normalizados de acordo com os coeficientes respectivos $\left(C_{R}\right.$ e $\left.C_{S}\right)$. Recentemente, Sanders $(11,12,13)$ procurou clarificar a controvérsia entre a preponderância das teorias propulsivas baseadas na sustentação hidrodinâmica e resistência propulsiva, utilizando como referência os valores dos coeficientes $\left(C_{R}\right.$ e $\left.C_{S}\right)$ obtidos em experiências efectuadas no Instituto de Investigação Hidráulica (Iowa, EUA), determinados para a amplitude total de ângulos de orientação e ataque possíveis, definidos de acordo com a convenção proposta por Schleihauf (15).

Existem, no entanto, muitas dúvidas acerca da fiabilidade do cálculo de forças gerado tendo como base a aplicação dos procedimentos experimentais. Dois tipos de problemas podem ocorrer com a aproximação experimental (11):

1. Problemas relacionados com o setup experimental, especificamente:

i) Erros derivados do processo de digitalização. Para calcular as forças produzidas pela mão existe a necessidade de digitalizar pequenas referências na mão, para definir o seu plano. A mão é tradicionalmente difícil de digitalizar de uma forma precisa, uma vez que os pontos anatómicos são muito pequenos e apresentam-se muito próximos, sendo o processo ainda dificultado pelos distúrbios gerados pela refracção da água e pela presença de bolhas de ar na água $(10,11)$.

ii) Erros derivados da distorção decorrente das técnicas de registo de imagem subaquáticas. Para se obterem boas imagens tridimensionais para o cálculo das forças geradas pela mão, é necessário um setup com múltiplas câmaras subaquáticas, facto que gera problemas na análise decorrente da turbulência gerada em torno dos segmentos (11).

iii) Erros derivados do cálculo do $C_{R}$ e $C_{S}$. Apenas alguns estudos publicaram resultados dos valores do $\mathrm{C}_{\mathrm{R}}$ e $\mathrm{C}_{\mathrm{S}}$ para a mão e antebraço $(1,2,11,15,16)$. No entanto, mesmo nestes poucos estudos efectuados, foram obser vados diferenças na magnitude dos coeficientes $(10 \%$ a $17 \%)$, principalmente decorrentes das diferentes metodologias usadas para a sua quantificação. Numa, quase, replicação do trabalho de Schleihauf, Berger et al. (2) calcularam valores de força, sob regime permanente, $17 \%$ mais baixos do que os valores de força medidos experimentalmente, utilizando para o efeito o measure active drag system (MAD), sugerindo a existência de mecanismos de instabilidade significativos no regime de fluxo. No final da década de 90, Berger, Hollander \& Groot (3), utilizando a mesma metodologia comparativa, descreveram diferenças de aproximadamente $10 \%$ entre os valores calculados e medidos $\left(\mathrm{F}_{\mathrm{MAD}}=34 \mathrm{~N}\right.$; $\mathrm{F}_{\text {Schlei }}=30 \mathrm{~N}$ ). 
2. Problemas associados com pressupostos incorrectos: $\mathrm{O}$ método para a quantificação da força produzida pela mão/antebraço mediante aproximações indirectas baseia-se no pressuposto de que existe um regime de fluxo permanente, resultando em forças estáveis para cada velocidade e orientação dos segmentos propulsivos. De facto, os coeficientes $\left(\mathrm{C}_{\mathrm{R}}\right.$ e $\left.\mathrm{C}_{\mathrm{S}}\right)$ são geralmente determinados sob condições de velocidade, direcção e orientação constantes dos segmentos propulsivos. No entanto, a realidade não é esta. A formação e separação de vórtices traduzem alguma instabilidade no fluxo e, consequentemente, na produção de forças, mesmo para casos de direcção e orientações dos segmentos constantes. O trabalho recente de Sanders (11) produziu coeficientes adicionais para serem aplicados às acelerações lineares na direcção do deslocamento, neste caso da mão, integrando a instabilidade decorrente do regime de fluxo. Recentemente Toussaint, Berg \& Beek (18) reafirmaram a possibilidade de o cálculo quase estático das forças não reflectir perfeitamente a força propulsiva obser vada e a eficiência propulsiva durante o nado na técnica de crol, sugerindo mesmo a existência de significativos mecanismos de instabilidade do fluxo. Todos estes problemas sugerem ser limitado o potencial aplicativo dos coeficientes de força existentes (indirectos) para modelos de mão/antebraço. Uma alternativa à prática experimental comple xa, morosa e onerosa, é a aplicação de técnicas numéricas de DCF, não só nos desportos aquáticos em geral e na Natação em particular $(5,6)$, mas também em aplicações médicas $(4,9)$.

O estudo de Bixler \& Schloder (6) de DCF foi realizado, quer para condições de fluxo em regime permanente, quer não permanente (acelerado), sob a superfície externa de um disco com uma área similar à área típica da mão de um nadador. Diferentes simulações com variação dos valores de velocidade inicial e aceleração foram escolhidas, no limiar das condições verificadas no nado real, fundamentalmente durante a acção lateral interior e acção ascendente da braçada de crol. De acordo com os resultados obtidos, a aceleração da mão pode aumentar em cerca de $24 \%$ a força propulsiva, quando em regime permanente de fluxo. Desde então, apenas mais um estudo (5) foi realizado no qual o principal objectivo foi o cálculo dos coeficientes $\left(C_{R}\right.$ e $\left.C_{S}\right)$ mediante a aplicação de uma análise bidimensional da mão/antebraço. As simulações efectuadas permitiram confirmar a importância utilização da DCF na Natação, assim como a confirmação, por análise comparativa, dos valores dos coeficientes computacionais obtidos.

Desta forma, é duplo o objectivo do presente estudo: a) dar continuidade à utilização da DCF como uma nova metodologia na investigação na Natação; b) aplicar a DCF na determinação da contribuição relativa dos coeficientes de sustentação e resistência $\left(\mathrm{C}_{\mathrm{S}}\right.$ e $C_{R}$ ) resultantes da resolução numérica bidimensional do fluxo externo permanente de três modelos da mão/antebraço do nadador.

O presente estudo representa uma etapa adicional na aplicação dos métodos de resolução numérica na Natação, uma vez que tentamos resolver alguns dos aspectos críticos dos trabalhos efectuados neste domínio $(5,6)$, fundamentalmente:

i) Pelo cálculo bidimensional da força, e coeficientes $\left(C_{R}\right.$ e $\left.C_{S}\right)$, baseado numa geometria real de um modelo de mão/antebraço e não de um disco com área similar;

ii) Pelo uso de uma malha mais comple xa com maior resolução, e a eliminação do ponto de singularidade: (a) pelo uso de uma secção adicional ( $3^{a}$ ) na construção do modelo da mão e antebraço; (b) pelo uso de uma geometria trapezóide na definição da malha; iii) suavização do contorno do modelo da mão e antebraço, através da eliminação da rugosidade responsável pela perturbação do fluxo externo do modelo da mão/antebraço.

\section{MATERIAL E MÉTODOS}

A Dinâmica Computacional de Fluidos (DCF) consiste na realização de simulações numéricas, através de cálculos iterativos que resolvem equações predefinidas, que permitem obter os resultados perseguidos. O modelo matemático é aplicado ao escoamento do fluido. As forças geradas pela mão/antebraço de um nadador, resistência $\left(F_{d}\right)$ e sustentação $\left(F_{1}\right)$, são função da velocidade do fluido $\left(\mathrm{V}_{\mathrm{x}}\right.$ e $\left.\mathrm{V}_{\mathrm{y}}\right)$, de acordo com a expressão matemática $n^{\circ} 1$ :

$V=\sqrt{V_{x}^{2}+V_{y}^{2}}$ 
Considerando o facto de o modelo da mão/antebraço estudado ser fixo e tendo em consideração a área da projecção do modelo no plano perpendicular à força para diferentes ângulos de ataque $\left(\theta=0^{\circ}, 90^{\circ}, 180^{\circ}\right)$ - $\left(\mathrm{A}_{1}=0,0107 \mathrm{~m}^{2}, \mathrm{~A}_{2}=0,0191 \mathrm{~m}^{2}, \mathrm{~A}_{3}=0,0103 \mathrm{~m}^{2}\right)$ - e a densidade do fluido $(\rho)$, estas forças foram calculadas através das seguintes equações:

$F_{S}=C_{S} \cdot \frac{1}{2} \cdot \rho \cdot V^{2} \cdot A_{n=1,2,3}$

$F_{l}=C_{l} \cdot \frac{1}{2} \cdot \rho \cdot V^{2} \cdot A_{n=1,2,3}$

\section{Modelo matemático}

Num determinado domínio, o modelo matemático (Standard $\mathrm{k}-\varepsilon$ ) substitui as equações de Navier Stok es por expressões algébricas que foram resolvidas por cálculos iterativos. O domínio consiste numa malha bidimensional criada em torno do modelo da mão/antebraço, que simula o escoamento do fluido. Seguidamente são consideradas as propriedades mecânicas do fluido, as características do escoamento, as relações matemáticas que relacionam a turbulência e as condições de fronteira.

Nesta secção, os sistemas de equações baseado no modelo $\mathrm{k}-\varepsilon$ são apresentados, assim como o método de resolução usando o algoritmo AMG (Algebraic Multi-Grid). As equações de Reynolds para fluidos incompressíveis, assim como as equações de Navier Stok es com o modelo standard $k-\varepsilon$ model foram implementadas no código Fluent. De acordo com estas variáveis, as equações da continuidade do movimento, da conser vação do momento e turbulência, da dissipação e conser vação de energia $(k-\varepsilon)$ para um fluido incompressível em coordenadas cartesianas foram escritas de forma conser vativa, de acordo com as seguintes expressões:

$$
\operatorname{div} U=0
$$

$$
\frac{\partial U}{\partial t} \pm U . \nabla U+\nabla p \pm \nabla\left(v+c \mu \frac{k^{2}}{\varepsilon}\right)\left(\nabla U+\nabla U^{t}\right)=0
$$

$$
\begin{aligned}
& \frac{\partial \mathrm{pk}}{\partial \mathrm{t}}+\frac{\partial \rho \mathrm{V}_{\mathrm{x}} \mathrm{k}}{\partial \mathrm{x}}+\frac{\partial \rho \mathrm{V}_{\mathrm{y}} \mathrm{k}}{\partial \mathrm{y}}=\frac{\partial\left(\frac{\mu_{\mathrm{t}} \partial \mathrm{k}}{\sigma_{\mathrm{k}} \partial \mathrm{x}}\right)}{\partial \mathrm{x}}+\frac{\partial\left(\frac{\mu_{\mathrm{t}} \partial \mathrm{k}}{\sigma_{\mathrm{k}} \partial \mathrm{y}}\right)}{\partial \mathrm{y}}+\mu_{\mathrm{t}} \phi-\rho \varepsilon \\
& \frac{\partial \mathrm{pk}}{\partial \mathrm{t}}+\frac{\partial \rho \mathrm{V}_{\mathrm{x}} \varepsilon}{\partial \mathrm{x}}+\frac{\partial \rho \mathrm{V}_{\mathrm{y}} \varepsilon}{\partial \mathrm{y}}=\frac{\partial\left(\frac{\mu_{\mathrm{t}} \partial \varepsilon}{\sigma_{\varepsilon} \partial \mathrm{x}}\right)}{\partial \mathrm{x}}+\frac{\partial\left(\frac{\mathrm{t} \partial \varepsilon}{\sigma_{\varepsilon} \partial \mathrm{y}}\right)}{\partial \mathrm{y}}+\mu_{\mathrm{t}} \frac{\varepsilon}{\mathrm{k}} \phi-\mathrm{C}_{2} \frac{\rho \varepsilon}{k}
\end{aligned}
$$

Nas expressões (4) a (7), $k$ é a energia cinética de turbulência, $\varepsilon$ é a taxa de dissipação de energia cinética de turbulência, $V_{x}$ e $V_{y}$ representam a componente horizontal $(x)$ e vertical (y) da velocidade $U$; $\mu_{\mathrm{t}}$ é a viscosidade turbulenta e $\rho$ representa a densidade do fluido; $t$ é a temperatura do fluido; $\mathrm{x}$ e $\mathrm{y}$ são, respectivamente, as coordenadas horizontais $e$

\begin{tabular}{|c|c|}
\hline Cons. & Standard k- $\varepsilon$ \\
\hline$C_{2}$ & 1,92 \\
\hline$C_{u}$ & 0,09 \\
\hline$\sigma_{\varepsilon}$ & 1,30 \\
\hline$\sigma_{k}$ & 1,00 \\
\hline
\end{tabular}
verticais. As outras constantes estão representadas no Quadro 1.

Quadro 1: Constantes do modelo $k-\varepsilon$ modificado.

\section{Aplicação}

Considerando o facto do presente estudo ter sido baseado numa análise bidimensional, foram simulados três modelos de mão/antebraço com três planos diferentes. Um modelo frontal $\left(\theta=90^{\circ}, \Phi=0^{\circ}\right)$ e dois modelos laterais, um tendo o dedo mindinho como bordo de ataque $\left(\theta=0^{\circ}\right.$ e $\left.\Phi=0^{\circ}\right)$ e o outro tendo o dedo polegar como bordo de ataque $\left(\theta=180^{\circ}\right.$ e $\left.\Phi=0^{\circ}\right)$.

Para a definição das diferentes orientações do fluxo permanente em torno dos modelos foi utilizada a convenção aceite pela comunidade científica (15). A Figura 1 ilustra os dois planos:

- O plano longitudinal $(\Phi)$ ou ângulo de orientação, que representa a rotação do plano coronal do corpo humano;

- O plano radial $(\theta)$, ou ângulo de ataque, que representa o ângulo gerado pela linha de fluxo, relativamente à posição do modelo ou o plano transverso. 


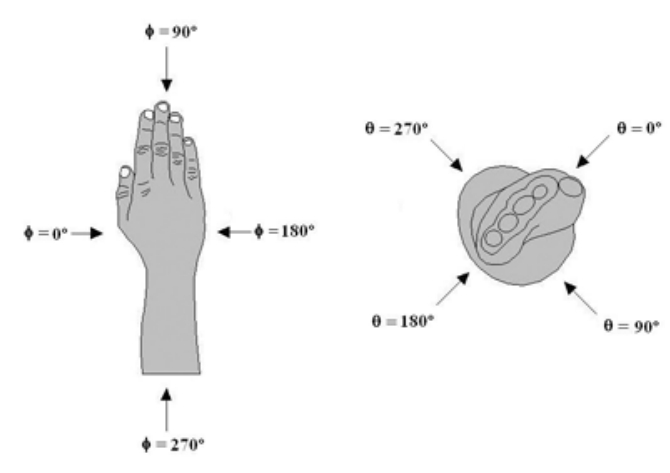

Figura 1: Ângulos de orientação e de ataque dos modelos.

Os modelos utilizados nas simulações foram criados em $\mathrm{CAD}$ e foram baseados nas dimensões reais de uma mão/antebraço direito de um ser humano adulto. Os modelos foram subdivididos em três segmentos: a mão $(190 \mathrm{~mm})$, o antebraço $(210 \mathrm{~mm})$ e a base $(85 \mathrm{~mm})$, tal como pode ser obser vado na figura 2 .

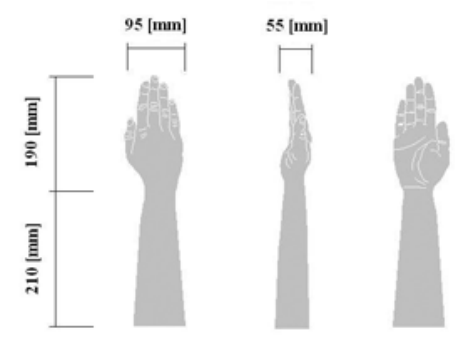

Figura 2: Dimensões do modelo.

O principal objectivo da base, como terceira secção, foi o de eliminar o ponto de singularidade obtido em simulações prévias, no qual elevadas pressões foram identificadas. No entanto, a existência da base, como terceira secção, não interfere com a medição das forças resultantes nos três modelos estudados.

Para que a visualização do modelo matemático seja perceptível, a Figura 3 mostra-nos as diversas condições de fronteira inerentes ao problema: a força gravítica a que o fluido e o corpo estão sujeitos; as diversas velocidades a que as simulações foram realizadas, bem como as dimensões do domínio. De notar que as dimensões deste são bastante superiores às dimensões do modelo mão/antebraço, de forma a garantir que não existam perturbações nos limites do domínio que influam no escoamento sobre o modelo, caracterizando, tanto quanto possível, a submersão da mão/antebraço e livre escoamento do fluido.

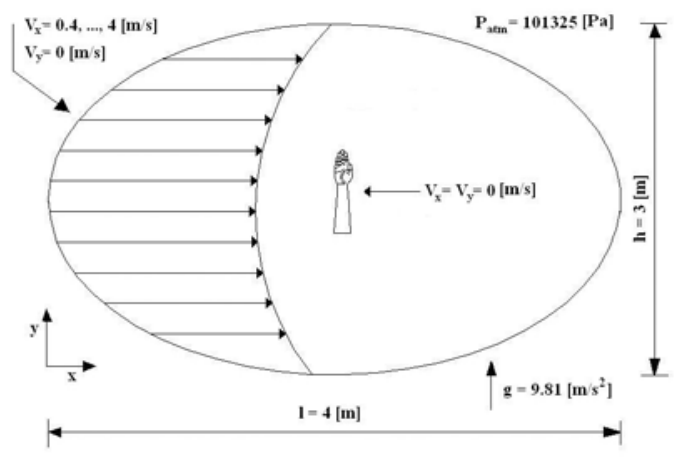

Figura 3: Condições de fronteira aplicadas ao modelo computacional.

A totalidade do domínio foi construída com uma malha de 400 mil elementos trapezoidais de quatro nós cada. As condições de fronteira, para as simulações em regime de fluxo permanente foram as seguintes:

- No lado esquerdo do domínio, a componente horizontal inicial da velocidade $\left(0,50 \mathrm{~m} . \mathrm{s}^{-1}\right)$ sofreu um incremento de 0,50 (m.s $\left.{ }^{-1}\right)$ até aos 4,00 (m.s $\left.{ }^{-1}\right)$. A componente vertical da velocidade, foi sempre igual a zero;

- No lado direito do domínio, a pressão foi igual a 1 atm, pré-requisito formal para não permitir $o$ refluxo do fluido;

- Em torno do modelo da mão/antebraço, as duas componentes da velocidade foram consideradas nulas, possibilitando a condição de não descolamento do fluido relativamente ao modelo.

Foi também considerada a acção da força de gravidade $\left(a_{\mathrm{g}}=9,81 \mathrm{~m} \cdot \mathrm{s}^{-2}\right)$, assim como a percentagem de turbulência de $1,00 \%$ com $0,10 \mathrm{~m}$ de comprimento. A água foi o fluido considerado, incompressível, com um valor de densidade $\left(\rho=996,60 \times 10 \quad-9 \mathrm{~kg} \cdot \mathrm{mm}^{-3}\right)$ e viscosidade determinados $\left(\mu=8,57 \times 10 \quad-7\right.$ kg.mm $\left.{ }^{-1} \cdot \mathrm{s}^{-1}\right)$.

As simulações foram realizadas tendo em conta somente a condição de regime permanente, isto é, o escoamento do fluido é constante e livre de qualquer 
aceleração. Como o modelo em causa compreende somente duas dimensões, a possibilidade do estudo da infinidade de orientações que a mão/antebraço poderá ter em relação ao fluido foi limitada a três direcções $\left(\theta=0^{\circ}, \quad \theta=90^{\circ}\right.$ e $\left.\theta=180^{\circ}\right)$.

Sob estas condições, os valores dos coeficientes de sustentação e de resistência hidrodinâmicas $\left(\mathrm{C}_{\mathrm{S}} \mathrm{e}\right.$ $\mathrm{C}_{\mathrm{R}}$ ) foram calculados pela aplicação das equações (1) e (2) e pela integração dos valores de pressão em torno dos modelos da mão/antebraço. O integral da superfície limitada pela pressão foi expressa pela seguinte equação.:

$$
F=\iint_{S} p d s
$$

\section{RESULTADOS}

Nas figuras 4 a 6 apresentamos as três curvas de pressão correspondentes a cada um dos modelos de orientação estudados. Como se pode constatar, a pressão permanece constante até ao final da superfície das mãos e, depois, decresce em quatro patamares, cada um representando as depressões correspondentes ao comprimento de cada um dos dedos. Finalmente, a pressão decresce para valores negativos correspondente à face posterior do modelo.

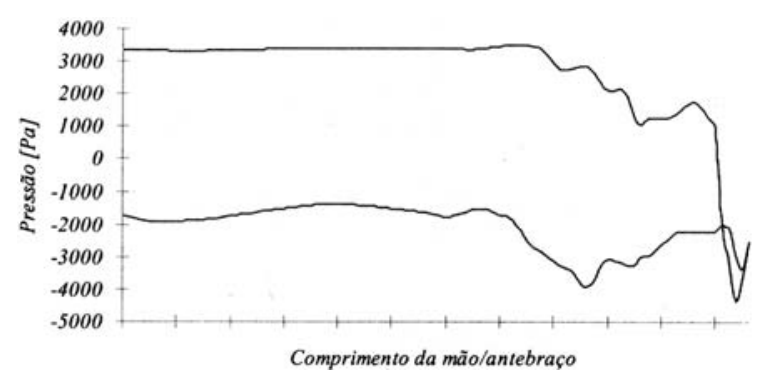

Figura 4: Distribuição da pressão em torno do modelo da mão/antebraço $\left(\theta=0^{\circ}\right.$ e $\left.\Phi=0^{\circ}\right)$.

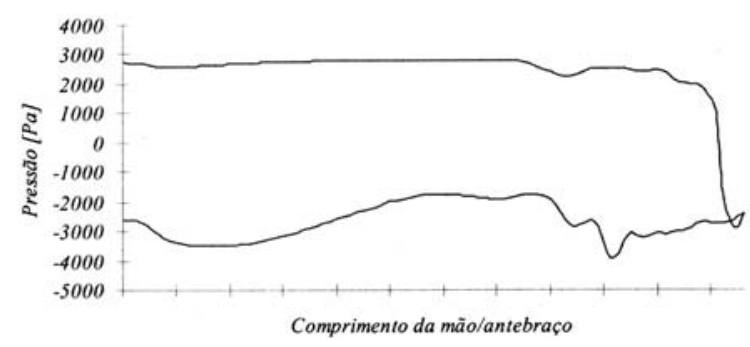

Figura 5: Distribuição da pressão em torno do modelo da mão/antebraço $\left(\theta=90^{\circ}\right.$ e $\left.\Phi=0^{\circ}\right)$.

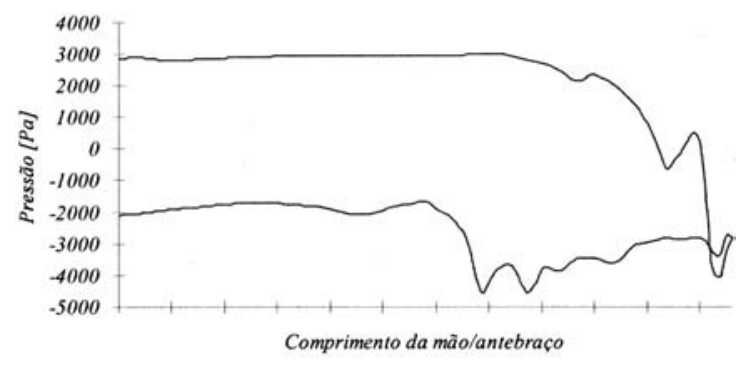

Figura 6: Distribuição da pressão em torno do modelo da mão/antebraço $\left(\theta=180^{\circ}\right.$ e $\left.\Phi=0^{\circ}\right)$.

Quadro 2: Valores máximos de CS, CR e força de resistência hidrodinâmica obtidos para o fluxo em regime permanente para os três modelos estudados (Vmão/antebraço=2 m.s-1).

\begin{tabular}{l|c|c|c} 
Ângulo de ataque & $C_{R}$ & $C_{S}$ & Força Resistência $(\mathrm{N})$ \\
\hline$\theta=0^{\circ}$ & 0,27 & 0,15 & 5,46 \\
\hline$\theta=90^{\circ}$ & 1,16 & 0,02 & 44,42 \\
\hline$\theta=180^{\circ}$ & 0,27 & 0,11 & 5,67
\end{tabular}

No quadro 2 podemos visualizar as forças e coeficientes registados para os diferentes ângulos de ataque, para uma velocidade de 2,00 m.s ${ }^{-1}$.

De acordo com os resultados obtidos, os valores do $\mathrm{C}_{\mathrm{R}}$ para a mão/antebraço, nos três modelos estudados, foram superiores aos obtidos para o $\mathrm{C}_{\mathrm{S}}$. $\mathrm{O}$ valor máximo de $C_{R}$ foi obtido para o modelo com um ângulo de ataque de $90^{\circ}\left(C_{R}=1,16\right)$ e os valores mínimos foram obtidos para os modelos com um ângulo de ataque de $0^{\circ}$ e $180^{\circ}$. 
$\mathrm{O} \mathrm{C}_{\mathrm{S}}$ permaneceu constante e quase nulo, no entanto, o valor máximo para $\mathrm{C}_{\mathrm{S}}$ foi obtido no modelo com um ângulo de ataque de $0^{\circ}$, i.e., tendo como bordo de ataque do segmento o dedo mindinho. Para um determinado ângulo de ataque, os valores dos coeficientes de força, foram praticamente constantes para toda a amplitude de velocidades estudadas.
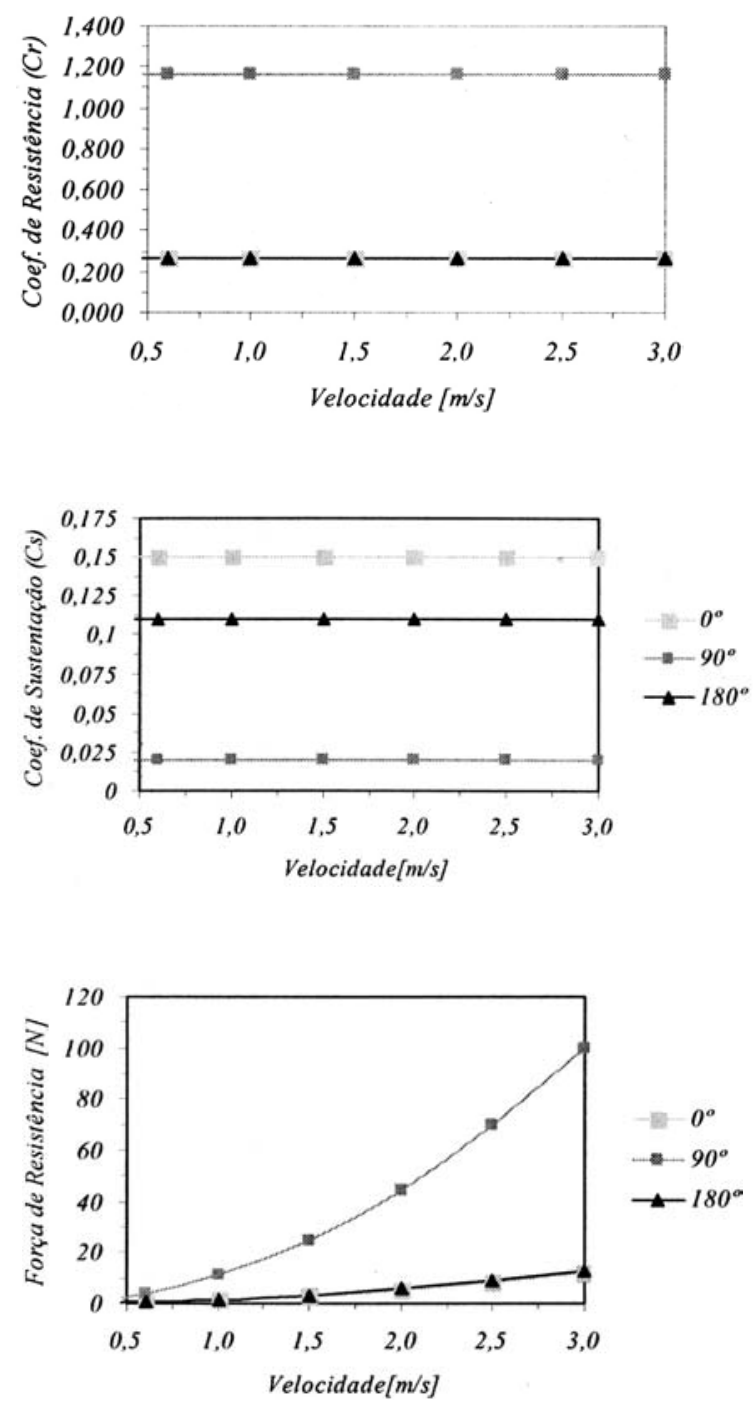

Figura 7: Representações gráficas, em função da velocidade de escoamento, da força de resistência e dos coeficientes de força de resistência (CR) e de sustentação (CS) hidrodinâmicas, para cada uma das três diferentes orientações.
$\mathrm{Na}$ figura 7 visualizam-se três gráficos construídos através dos valores registados nas diversas simulações. Como podemos verificar, os coeficientes CR e CL são praticamente constantes, não variando com o incremento da velocidade. O mesmo não se verifica com a força de propulsão, pois esta é função do quadrado da velocidade. Logo, o seu crescimento equivale a uma curva ascendente, sendo quase constante para velocidades até 1 (m.s $\left.{ }^{-1}\right)$ e crescendo exponencialmente até um máximo de 44,47 (N) para um ângulo de $90^{\circ}$ e $\left(2,00\right.$ m.s $\left.{ }^{-1}\right)$.

Como a força propulsiva é uma função da sustentação e da resistência, e visto estas variarem substancialmente de ângulo para ângulo, podemos afirmar que a contribuição da resistência na geração de força propulsiva para um ângulo de $90^{\circ}$ é máxima, sendo que os valores de sustentação são mínimos ou praticamente nulos, contribuindo de forma reduzida para o aumento na força propulsiva. Da mesma forma e para os restantes ângulos em estudo, os valores de resistência e sustentação são próximos entre si e de valores bastante baixos. Como a área de projecção para os ângulos de $0^{\circ}$ e $180^{\circ}$ é inferior à área de projecção para $90^{\circ}$ e sabendo que a força propulsiva está directamente relacionada com estas áreas, é lógico que os valores de força sejam máximos para ângulos próximos dos $90^{\circ}$.

\section{DISCUSSÃO}

O resultado dos valores de pressão demonstrou que o modelo com uma orientação directamente oposta ao escoamento do fluido apresentou os valores mais elevados do $C_{R}$. No bordo de fuga do modelo, pode obser var-se uma separação significativa do escoamento na camada limite, formando uma depressão e produção de vórtices. Estes resultados eram esperados e revelaram o diferencial de pressões que provoca a propulsão do nadador .

Com base nos resultados obtidos nas diferentes simulações efectuadas em regime permanente, poderemos assumir que a DCF pode ser considerada como uma nova e interessante metodologia para o cálculo das forças hidrodinâmicas em Natação, fornecendo, também, informações adicionais para a definição de novas interpretações das técnicas de nado. Os valores de força calculados pela mão/antebraço foram decompostos no $C_{R}$ e $C_{S}$. A magnitude 
dos valores de força resistência e de sustentação obtidos estão na dependência directa da orientação dos três modelos estudados. Apesar do facto recorrente de o $C_{R}$ e o $C_{S}$ representarem a já tradicional aproximação e paradigma de análise da propulsão em Natação no conte xto de estabilidade dos movimentos, torna-se, no entanto, determinante a avaliação da fiabilidade inicial da DCF pela comparação com publicações prévias, experimentais e computacionais, nas quais o $C_{R}$ e $C_{S}$ foram calculados no mesmo regime de fluxo (permanente).

Sob a condição de fluxo em regime permanente, os resultados obtidos para os coeficientes $\left(C_{R}\right.$ e $\left.C_{S}\right)$ são similares aos valores obtidos em estudos experimentais $(1,12,13,15,19)$, factor importante para a validação metodológica da DCF, fornecendo também condições de aceitação iniciais para a análise das forças hidrodinâmicas produzidas sob condições de instabilidade do fluxo.

Para os três diferentes modelos de orientação e para a totalidade das amplitudes estudadas, o $C_{R}$ e o $C_{S}$ permaneceram praticamente constantes, demonstrando uma evolução gráfica num padrão linear. Resultados similares foram obser vados em estudos de DCF (6). Apesar do comportamento similar para os três modelos estudados, o modelo com um $\theta=90^{\circ}$ evidenciou valores superiores de $C_{R}$ e de força resistência propulsiva. Os outros dois modelos apresentaram resultados similares, mas com valores mais reduzidos, o que nos permite concluir pela não existência de fenómenos propulsivos de relevância sob estas duas condições de fluxo, quer para o $C_{R}$ quer para o $\mathrm{C}_{S}$.

No final da década de 70, Wood (19) testou um modelo de mão/antebraço num túnel de vento. A análise bidimensional efectuada foi em regime de fluxo permanente. Os valores obtidos permitiram concluir que, para ângulos de ataque próximos dos $90^{\circ}$, o $_{R}=1,10$, foi similar ao obtido no presente estudo. Este facto foi válido não só para o $\mathrm{C}_{\mathrm{R}}$ mas também para o $\mathrm{C}_{S}$.

No presente estudo, o $C_{R}$ obteve o valor mais elevado no modelo de mão/antebraço perpendicular à direcção do fluxo $\mathrm{O}$ mesmo resultado foi registado por Berger et al. (1) no qual os valores de força resistência aumentaram até um máximo verificado, nas mesmas condições de fluxo, idêntico aos verifi- cados neste estudo (ângulo de ataque de 90 ), apesar dos valores evidenciados por Berger et al. (1) terem sido calculados tendo como base o cálculo da área total, enquanto que os valores obtidos neste estudo foram calculados usando a área plana. Mais recentemente e usando dados experimentais dos coeficientes de $C_{R}$ e $C_{S}$, obtidos como resultado de experiências efectuadas num tanque de testagem no Instituto de Investigação Hidráulica (Iowa, EUA) e para a amplitude total e possível de ângulos de ataque e orientação, definidos de acordo com a convenção proposta por Schleihauf (15), Sanders (12, 13) concluiu que os valores mais elevados de força foram obtidos quando os ângulos de ataque eram próximos de $90^{\circ}$. Nesta orientação, tal como no presente estudo, a força gerada foi quase totalmente devida à resistência propulsiva. Outra conclusão importante foi produzida pelo estudo de Toussaint et al. (18). De acordo com estes autores, durante a maior parte da acção de membros superiores no trajecto subaquático, a direcção do fluxo foi inconsistente com a circulação em torno da mão e a sustentação gerada pode ser assumida, nestas condições de fluxo, de importância secundária ou acessória na produção de força propulsiva.

Somente dois estudos de DCF foram efectuados assumindo a Natação como domínio de aplicação. $\mathrm{Na}$ década de 90, Bixler \& Schloder (6) realizaram uma aproximação bidimensional com um disco com uma área similar à área da mão, obtendo valores do $\mathrm{C}_{\mathrm{R}}$ de 1.16, igual aos valores obtidos no presente estudo. O mesmo grupo de investigação (5), calculou os valores do $C_{R}$ e $C_{S}$ com base na DCF usando um modelo real da mão/antebraço em vez de um disco. Os valores apresentados para o $C_{R}$ foram de aproximadamente 1,00 .

No entanto, ambas as simulações, regime permanente e não permanente, foram efectuadas com modelos tridimensionais da mão/antebraço, apesar dos valores publicados apresentarem somente os resultantes dos cálculos bidimensionais. Estes valores foram, também, similares aos apresentados no presente estudo. $O$ erro na determinação do $C_{R}$ entre os dois estudos, foi de $14 \%$. No entanto, esta diferença pode ter origem nos seguintes factores:

i) No presente estudo foi utilizada uma malha adaptativa de 400 mil elementos trapezoidais de quatro 
nós, cada elemento, no sentido de alcançar a melhor definição possível dos contornos dos modelos da mão/antebraço. No estudo de Bixler \& Riewald (5) foi só utilizada uma malha com 215 mil elementos; ii) A eliminação do ponto de singularidade, no presente estudo, pelo uso da terceira seç̧ão na construção do modelo da mão/antebraço, não usado no outro estudo (5).

\section{CONCLUSÕES}

Sumariando, os resultados principais deste estudo permitem-nos concluir que: a) a Dinâmica Computacional de Fluidos, ao ser aplicada a escoamentos assumidos como típicos das técnicas de Natação, apresenta resultados coerentes e consistentes com os já disponíveis na literatura, pelo que poderá vir a assumir -se como uma nova aproximação metodológica importante para o cálculo das forces hidrodinâmicas na Natação; b) para os modelos limitados que foram estudados, os resultados da produção de força sugerem que a força de sustentação hidrodinâmica é de importância secundária, sob condições de escoamento permanente.

Como referência final, deveremos considerar este estudo como mais uma etapa na aplicação da DCF na investigação em Natação. Equaciona-se, no entanto, o problema de saber como é que a DCF poderá ser aplicada para analisar a produção de força na mão/antebraço, quando verdadeiros movimentos (geradores de instabilidade nos escoamentos) estão presentes. Relativamente a este tópico, os próximos estudos de DCF deverão incluir outros aspectos do movimento não equacionados no presente estudo, fundamentalmente as rotações multi-axiais e as acelerações positivas e negativas, o que provoca a necessidade de rodar a malha relativamente ao fluxo externo dos modelos com a alteração subsequente dos ângulos de ataque e orientação em todas as condições possíveis.

\author{
Abreviaturas \\ A - Área \\ F- Força \\ T - Temperatura \\ U - Velocidade \\ $\mathrm{V}$ - Velocidade \\ A - aceleração \\ $\mathrm{d}$ - Derivada \\ k - Coeficiente de energia cinética \\ $\mathrm{t}$ - Tempo \\ $\varphi$ - ângulo de orientação \\ $\mu$ - Viscosidade \\ $v$ - Viscosidade dinâmica \\ $\theta-\hat{A}$ Angulo de ataque \\ $\rho$-Densidade \\ $\psi$ - Ângulo de orientação \\ AMG - Algebric Multi Grid \\ $\mathrm{C}_{\mathrm{r}}$ - Coeficiente de Resistência \\ $\mathrm{Cs}_{1}$ - Coeficiente de Sustentação \\ $\mathrm{F}_{\mathrm{r}}$ - Força resistência \\ $\mathrm{F}_{\mathrm{s}}$ - Força de sustentação
}

\section{CORRESPONDÊNCIA}

António José Silva

Universidade de Trás-os-Montes e Alto Douro

Departamento de Ciências do Desporto

CIFOP

R. Dr. Manuel Cardona

5000 Vila Real

ajsilva@utad.pt 


\section{REFERÊNCIAS}

1. Berger, MAM; de Groot, G. \& Hollander, AP (1995). Hydrodynamic drag and lift forces on human hand arm models. Journal of Biomechanics 28(2)125-133.

2. Berger, MAM; Hollander, AP \& de Groot, G (1997). Technique and energy losses in front crawl swimming. Medicine \& Science in Sports \& Exercise 29(11) 1491-1498.

3. Berger, MAM; Hollander, AP \& de Groot, G (1999). Propulsive force in front crawl swimming. Journal of Sports Science 17, 97-105.

4. Berthier, B; Bouzebar, R; \& Legallais, L(2002). Blood flow patterns in an automatically realistic coronary vessel influence of three different reconstruction models. Journal of Biomechanics 35(10)1347-1356.

5. Bixler, BS \& Riewald, S (2002). Analysis of swimmer 's hand and arm in steady flow conditions using computational fluid dynamics. Journal of Biomechanics 35, 713-717.

6. Bixler, BS; Schloder, M (1996). Computational fluid dynamics: an analytical tool for the $21^{\text {st }}$ centur y swimming scientist. Journal of Swimming Research 11, 4-22.

7. Cappaert, J \& Rushall, BS (1994). Biomechanical analyses of Champion Swimmers. Spring Valley, CA: Sports Science Association.

8. Cappaert, J (1993). 1992 Olympic report Limited Circulation to all FINA Federations. Colorado Springs: United States Swimming.

9. Marshall, I; Shunzi, Z; Papathanasapoulu, P; Hoskins, P \& Xui YY (2004). MRI and CFD studies of pulsative flow in healthy and stenosed carotid bifurcation models. Journal of Biomechanics 37 (5), 679-687.

10. Payton, CJ \& Bartlett, RM (1995). Estimating propulsive forces in swimming from three dimensional data. Journal of Sports Sciences 13, 447-454.

11. Sanders, RH (1999). Hydrodynamic characteristics of a swimmer 's hand. Journal of Applied Biomechanics 15, 3-26.

12. Sanders, RH (1997a). Extending the «Schleihauf» model for estimating forces produced by a swimmers hand. In B.O. Eriksson, L. Gullstrand (Eds.) Proceedings of the XII FINA World Congress on Sports Medicine. Goteborg: Chalmers Reproser vice, 421-428.

13. Sanders, RH. (1997b). Hydrodynamic characteristics of a swimmer Hand with adducted thumb: implications for technique. In B.O. Eriksson, L. Gullstrand (Eds.) Proceedings of the XII FINA World Congress on Sports Medicine. Goteborg: Chalmers Reproser vice, 429-434.

14. Schleihauf, RE (1978). Swimming propulsion: A hydrodynamic analysis. In R.M. Ousley (Ed.) ASCA 1977 World Clinic Year Book. Florida: Ft. Lauderdale, 49-85.

15. Schleihauf, RE (1979). A hydrodynamic analysis of swimming propulsion. In J. Terauds, \& E.W. Bedingfield (Eds.) Swimming III. Baltimore: University Park Press, 70-109.

16. Schleihauf, RE; Gray, L. \& DeR ose, J (1983). Three-dimensional analysis of hand propulsion in the sprint front crawl strok e. In P. Hollander, P. Huijing, \& G. De Groot (Eds.) Biomechanics and medicine in swimming, 173-183.

17. Toussaint, HM (2000). An alternative fluid dynamic explanation for propulsion in front crawl swimming. Proceedings of the XVIII International Symposium on Biomechanics in Sports. Applied Program. Chinese University of Hong Kong, China, 96-103.

18. Toussaint, H; Den Berg, CV \& Beek, WJ (2002). Pumpedup propulsion during front crawl swimming. Medicine and Science in Sports Exercises 34, 314-319.
19. Wood, TC (1977). A fluid dynamic analysis of the propulsive potential of the hand and forearm in swimming. Master of Science Thesis, Dalhouise University Press, Halifax, NS. 